\title{
Innovative image geo-referencing tool for decision support in wildfire fighting
}

\author{
A. Losso, L. Corgnati \& G. Perona \\ Turin Polytechnic, Italy
}

\begin{abstract}
Forest fires prevention, monitoring and extinguishing operations in complex orography regions present dramatic problems related to hard environmental conditions, low population density and accessibility limitations affecting interventions activities and damage assessment. In the scientific landscape, there are many existing image geo-referencing tools. They are developed in order to rectify images, which come from fixed ground station and/or satellite platforms. Some of these use satellite images to geo-reference complex orography regions and ground images to geo-referencing plane orography regions by using Ground Control Point collections. The present work describes an innovative projective geo-referencing algorithm able to geo-reference complex orography regions using a fixed ground station images. Besides, it does not need the collection of Ground Control Points, which is a very hard task in complex orography environments. The system is an innovative image geo-referencing tool conceived for decision support aid for wildfire fighting in alpine regions. The system is operating within a monitoring system in the Piedmont area (Northwestern Italy) and operates on images sensed by a fixed monitoring network. The georeferencing software operates with a geometric and projective algorithm based on a Digital Elevation Model (DEM). The algorithm is built up of three main modules: sensors lens aberration correction, field-of-view localization on DEM, image geo-referencing. As said before, the system does not need any collection of Ground Control Points to rectify images. At the output of the system, each image pixel is linked to its geographical (Lat/Lon and UTM) coordinates. The final products are: a collection of scenario images sensed in the visible band; geo-referenced images with highlighted alarm pixels with overlaying DEM levels; topographic layers containing information to be used in case of intervention. Final images contain geo-referenced information about the sensors' field of view on the analysed area, hot-spot positions, helicopter landing spot
\end{abstract}


positions, water supply positions, intervention squad's localization and so on. When fire is detected, the system identifies fire latitude and longitude, indicates accessibility to a hot spot and puts in evidence territory characteristics and available resources. The algorithm is part of a more complex system in which it complements an integrated fire risk evaluation and monitoring system, in order to enhance early warning processes and intervention and to more efficiently manage damage assessment.

Keywords: forest fires, fire monitoring, rectification, geo-referencing, image processing, alpine regions.

\section{Introduction}

Forest fires fight is a critical issue to preserve our environmental heritage. It is necessary to develop systems able to enhance the intervention efficiency and the operators' safety in case of fires too.

The present work describes an innovative tool within a forest fires monitoring system located on complex orography areas where the fire risk is high [1]. Each monitoring station is equipped with commercial sensors able to acquire and process images sensed on several frequency bands as: visible, visible + Near Infrared (NIR) and Thermal Infrared (TIR).

The sensors are piloted by an engine, which permits to scan the area of interest.

The system is able to automatically generate wide shot format to be sent, through GPRS or satellite communications, to the central server station.

Geo-referencing tool assigns each pixel's image to its geographical coordinates using geometric and projective methods on DEM. In particular it is the final part of an integrated forest fires detection system. The integrated system is built up of several interpretation and detection algorithms. The algorithms identify an alarm on images in which pixels contain an event of fire as flame or smoke $[2,3]$. Therefore, in case of alarm, the fire detection output is processed by geo-referencing method which identifies the exact fire latitude and longitude. Moreover, topographic layers contains DEM's additional information as: camera field of view, helicopter landing spot's positions, water supply positions,

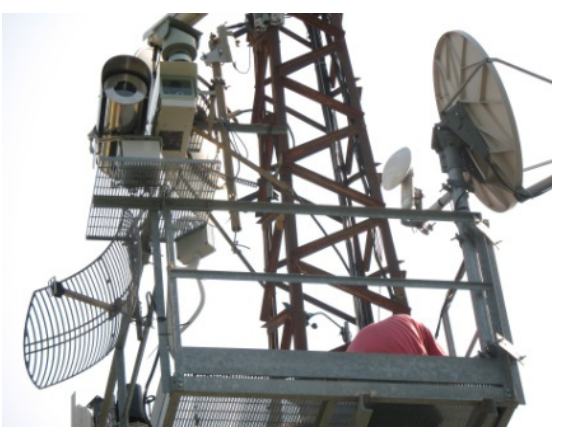

Figure 1: Monitoring station. 


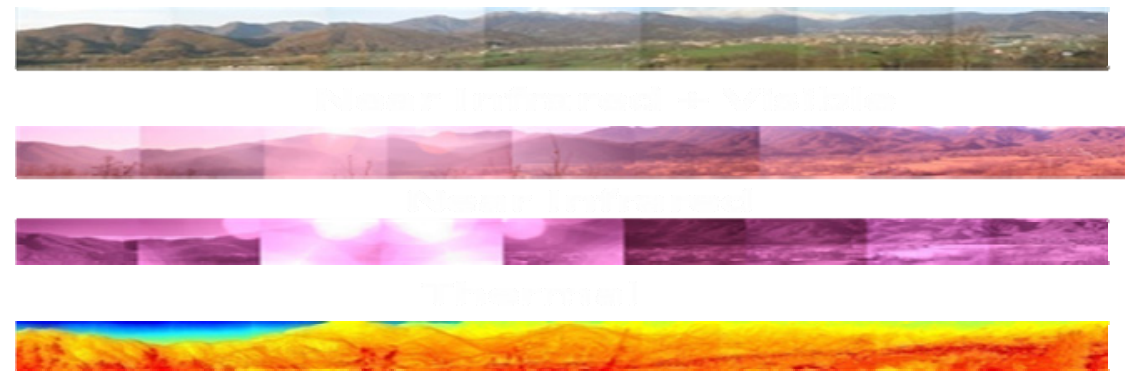

Figure 2: $\quad$ Example of frequency band sensed by monitoring station.

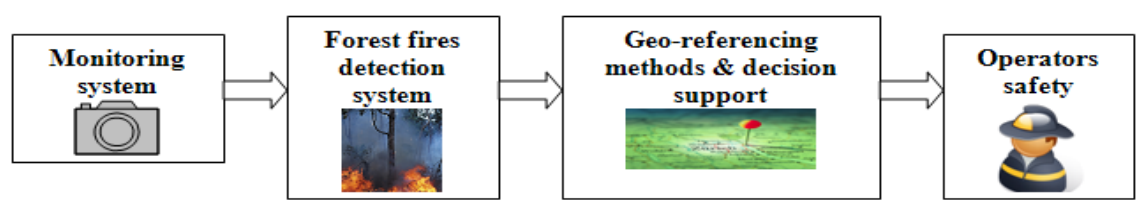

Figure 3: General block diagram of geo-referencing tool.

interventions squad's localization, service roads. If an alarm occurs, a complete set of geographical and logistic information is sent to the competent agencies.

Concerning early warning, the geographical position and the additional information are crucial for the early fire-extinguishing operations. In this paper we propose an innovative geo-referencing tool, which is able to generate georeferenced imagery created from standard images collected by low-cost commercial.

The main steps of the procedure are the following:

- Derivation of camera's aberrations correction. The aberrations are errors introduced by the camera lens.

- Digital Elevation Model. The method localizes the camera field of view on DEM.

- Geo-referencing tool. The method is able to link image's pixel to geographical UTM coordinates.

- Decision support. The method generates additional information layers related to the event of fire.

The software is implemented in Matlab(C) and provides a graphical user interface. Results point out a very high reliability and robustness in the decision support process. The geo-referencing system is a valuable aid in fire fight management: it allows involved agencies for an efficient resources handling (both logistic and human), for a finalized territory monitoring and for an intervention planning oriented to operators' safety. 


\section{Lens distortion}

Any photographic sensors, in particular commercial, could introduce a certain number of non-linear distortions due to aberrations in real lenses.

As a matter of fact, most frequent image imperfections (usually called aberrations) are: astigmatism, curvature of field, spherical aberration, geometric distortion and chromatic aberrations. In this work we take into account only the ones significant in our areas of work: the geometric distortion [4].

\subsection{Geometric distortion}

Geometric distortion is an aberration related to the position of points in the resulting image obtained from a real camera. It is crucial to consider distortion and aberration because they reduce the accuracy of measuring distances on the geo-referencing image so that they generate errors in the pixel position on image resulting in a displacement of image points with respect to their real positions.

Lens distortion is characterized by two main components called: Radial Distortion and Tangential Distortion.

\subsubsection{Radial distortion}

The geometric distortion affects the position of image points in the image. Using the distortion-free collinearity and some non-linear residual is possible to correct the errors caused by radial distortion. Two different types of distortion are possible, Barrel and Pin-cushion distortion.

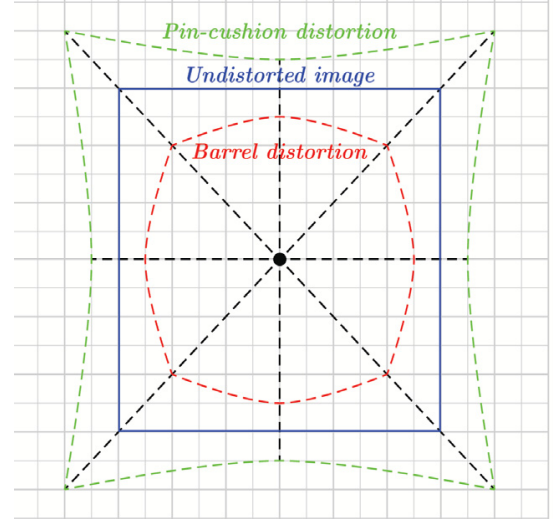

Figure 4: $\quad$ Barrel and pin-cushion distortion.

Basically, the barrel distortion causes the image points to crowd increasingly over the border and at the same time it causes the points close to the centre to spread on a radial direction.

The pin-cushion distortion presents opposite behaviour. It causes the image points to spread away when approaching the image border and at the same time it causes the image points to crowd when close to the image centre.

In order to correct geometric distortion a camera calibration tool is used. 
Figure 5 (b) (c) (d) shows the pixel behaviour after aberration corrections. In particular the arrows show how the lens calibration operates on the external pixel, according to Barrel and Pin-cushion distortions.

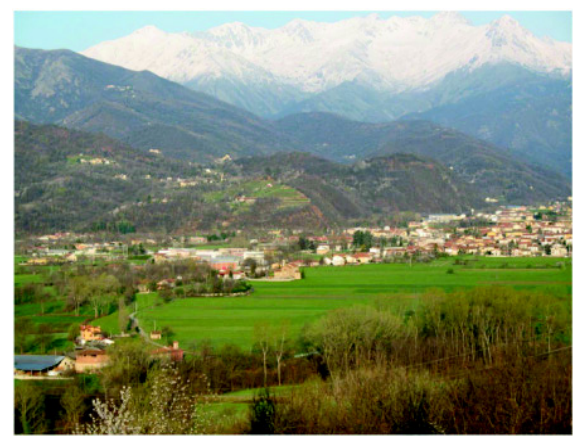

(a)

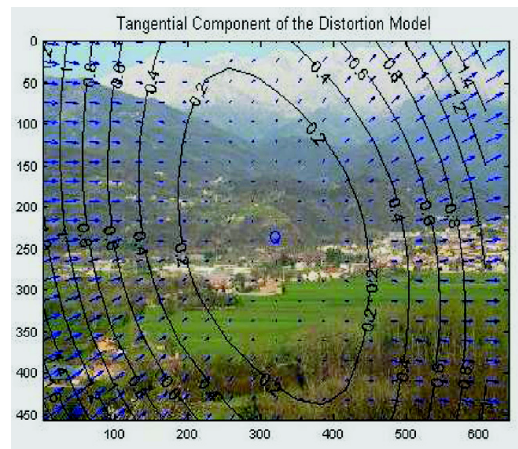

(c)

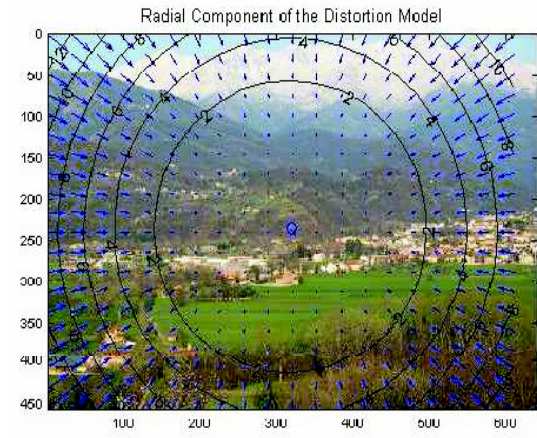

(b)

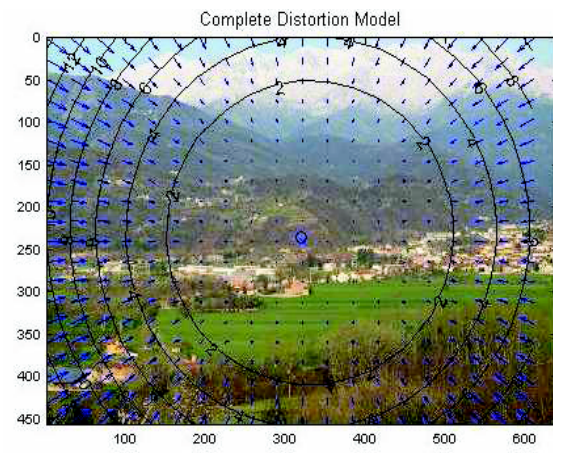

(d)

Figure 5: (a) Image affected by aberrations; (b) Radial correction; (c) Tangential correction; (d) Radial and Tangential correction.

\section{Digital elevation model (DEM) and field of view (FOV)}

The geo-referencing method does not use Ground Control Point collections to linked pixel in the geographical coordinates. In order to achieve our purpose we have to consider additional information and instruments. Concerning that, Digital Elevation Model is taken into account.

DEM is considered as a digital format territorial elevation distribution in which every single pixel is geo-referenced; in particular it is built up of raster format linking each pixel to its territorial elevation. Moreover, it is suited for Matlab@ applications. In our case study the region to be considered is Piedmont areas. 


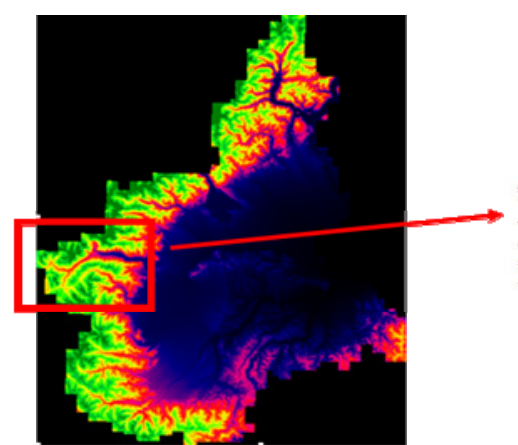

(a)

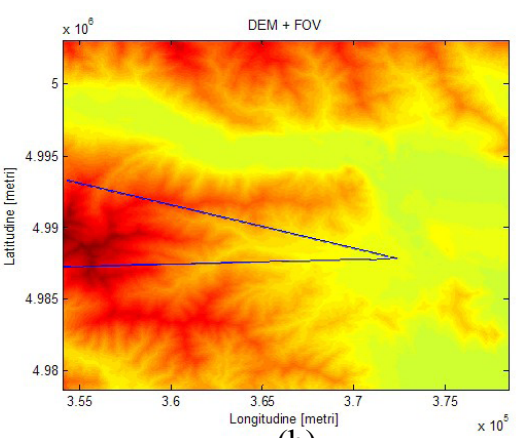

(b)

Figure 6: (a) DEM (b) Field of view on portion's DEM considered.

Digital elevation model is useful to build up the camera field of view. According to this point small area is take in consideration. The field of view is represented as a cone, whose vertex is the monitoring system location. For this purpose, view angles, tilt, pan, orientation with respect to North and horizon elevations are crucial components for the field of view construction [5].

\section{Geo-referencing tool and support decision}

Once image aberrations correction and camera sensor cone of view are set on DEM, the geo-referencing method starts up. It is based on projective and geometric methods in order to achieve the best geographical linking tradeoff $[6,7]$.

Elevation profile and geographical information are extracted from the cone of view on DEM; they represent the digital format view of images sensed by sensor. At the beginning, we just consider the horizon field of view and subdivide it into portions equals to horizon image resolution, as shown in Figure 7. For each portion the elevation profile is extracted, as shown Figure 8(a), the vertical field of view is subdivided into portion equals to vertical image resolution by using projective and geometric procedure, as shown in Figure 8(b). The selected portion is contained between two dotted lines, as shown in Figure 8(c).

Every single dotted line scans the vertical field of view from the bottom to the top a number of times equal to vertical image resolution. As it crosses the profile at least in one point, for each point it is possible to extract the relative geographical location, in particular the longitude location, fig 9. Basically, every line represents a certain vertical resolution value, linked to the longitude value. For example considering the first horizon and vertical scanning, if the first dotted line crosses the profile, the longitude value will be associated to the bottom left pixel's image and so on.

In order to find latitude component of the pixel just considered, we back up to the horizon field of view on DEM, fig 7(b). 


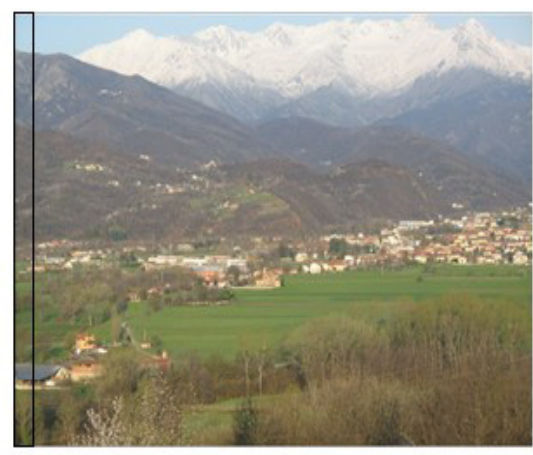

(a)

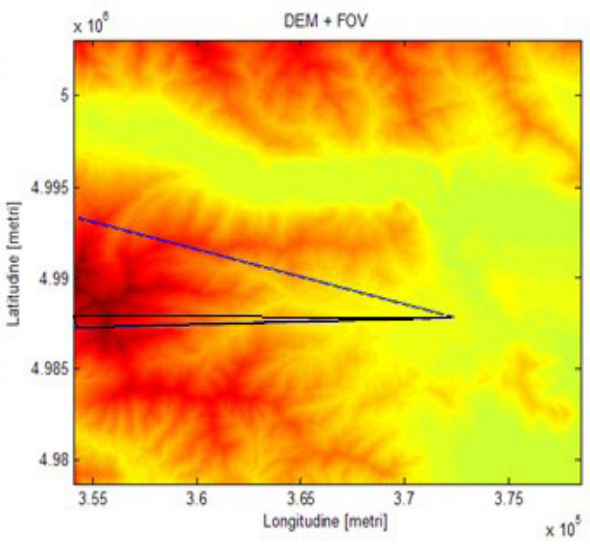

(b)

Figure 7: (a) Example of field of view portion considered on image; (b) Example of horizon field of view portion considered.

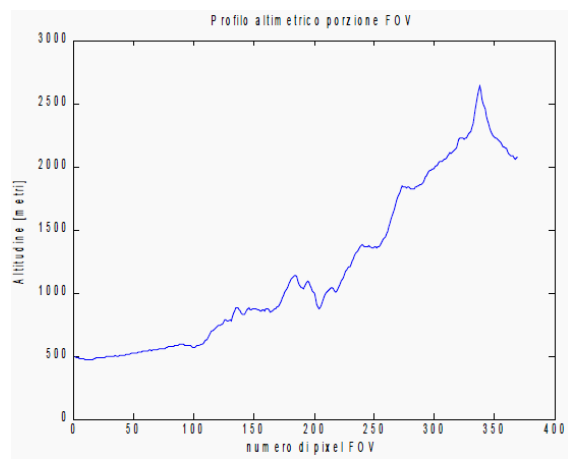

(a)

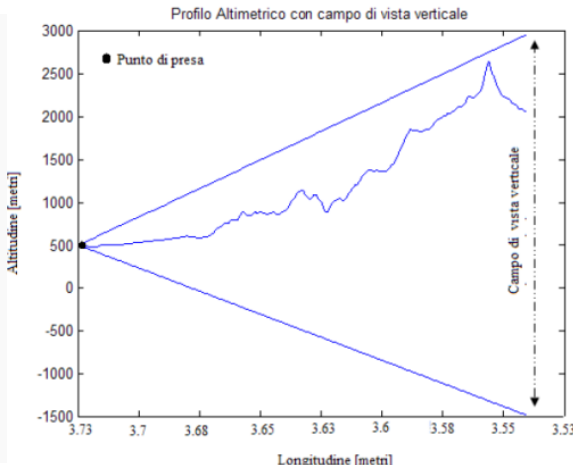

(b)

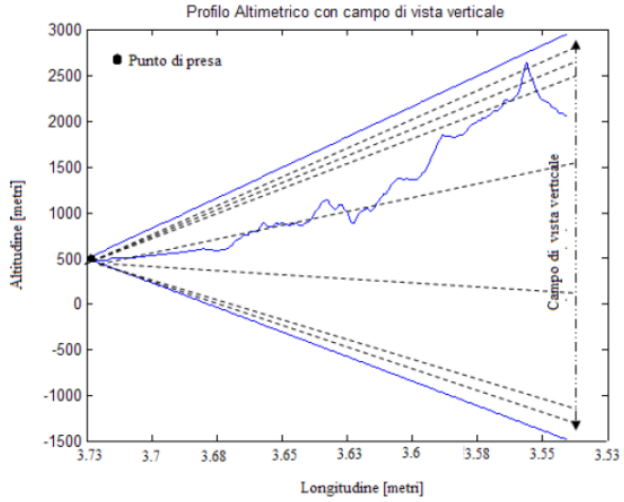

(c)

Figure 8: (a) Portion field of view profile; (b) Vertical camera field of view; (c) Dotted lines equals to vertical resolution. 
180 Modelling, Monitoring and Management of Forest Fires II

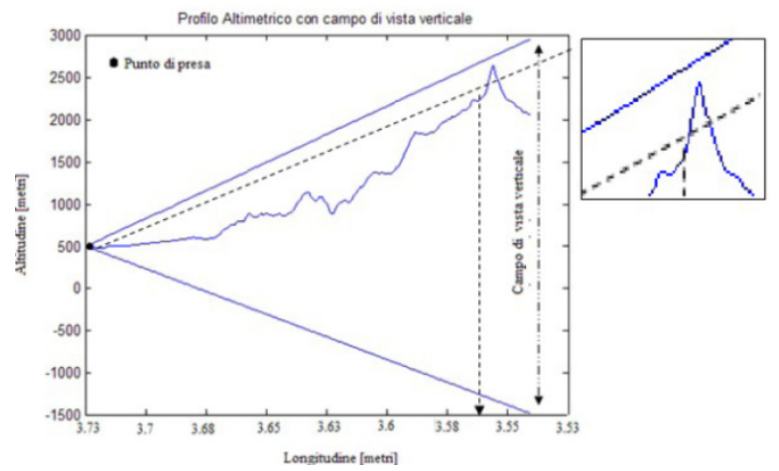

Figure 9: Dotted lines crosses the profile, determines longitude information.

At this point, knowing the longitude information, it is possible to determine the latitude information as shown on fig 10.

We trace a dotted line form to the longitude value until it crosses horizon field of view portion. The point determines the latitude value associated to the bottom left pixel, as mentioned in example.

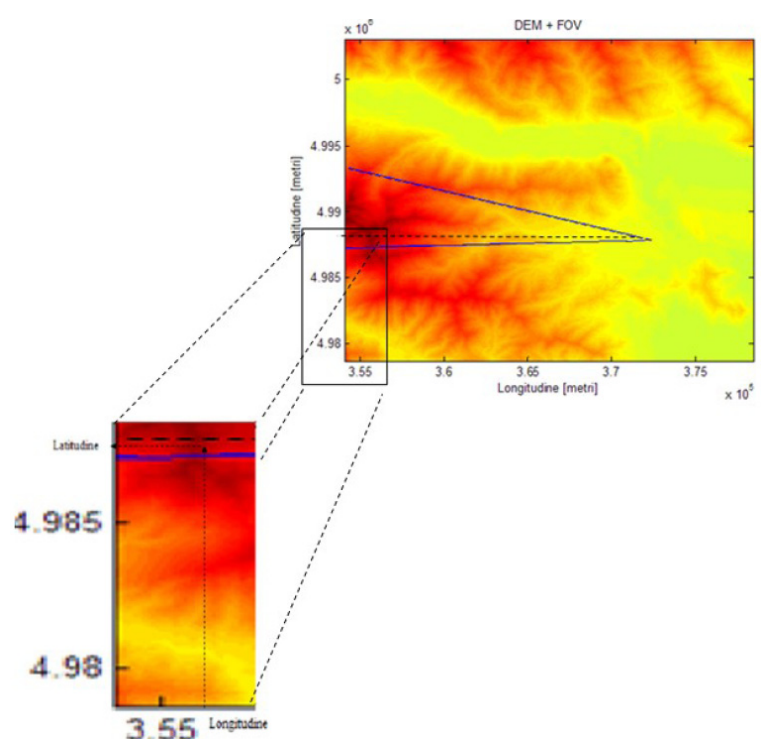

Figure 10: Determining the latitude component.

\subsection{Decision support}

In the case of a fire event, the forest fires detection algorithm identifies the pattern on the monitored scene. Geo-referencing tool is able to determine the exact geographical position of the event. At this point, it is possible to integrate additional information levels to be overlapped on DEM. The information levels 
are useful because they give additional tools to be used by operators. As an example, knowing the exactly geographical fire position it is possible to overlap on DEM logistic information, roads, helicopter landing, water supply positions, intervention squads, and so on. Moreover, all of them are geo-referenced and it is possible to choose them depending on the fire proximity.

\section{Preliminary test and results}

In order to test the algorithm, we built specific validation datasets made up of images taken from our test areas, located on Piedmont region. The main idea is to identify and mark some fixed points (FP) of known geographical positions within the images. For every single point, the real geographical coordinates have been checked using a GPS receiver in order to establish the mismatching between the real geographical coordinates and the results. As mentioned above, considering the image on fig. 5, we mark pixel coordinates, fig. 11, and the relative latitude and longitude are acquired by GPS receiver in the UTM geographical domain, tab. 1. For the best performance trade-off the image on fig. 5, has been converted in gray scale and masked on the areas where the fire risk is not significant, e.g. mountain snow cover.

The mismatches between real FP and geo-referencing method results are shown on fig. 12. The graph shows us the FP punctual difference and the avarage differences in latitude, longitude and distance in meters.

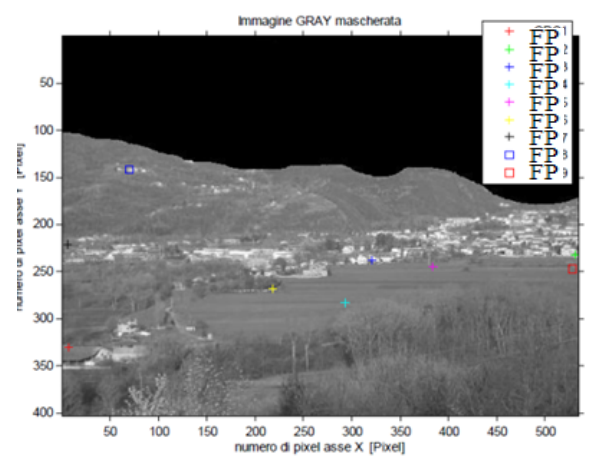

Figure 11: Image converted in grey scale, masked and marked with FP.

Table 1: $\quad$ FP pixel coordinates and the related true geographical coordinates.

\begin{tabular}{|c|c|c|c|c|c|c|c|c|c|}
\hline & FP1 & FP2 & FP3 & FP4 & FP5 & FP6 & FP7 & FP8 & FP9 \\
\hline $\begin{array}{c}\text { Pixel } \\
\text { coord. } \\
\text { X }\end{array}$ & 7 & 531 & 321 & 293 & 384 & 218 & 6 & 70 & 528 \\
Y & 330 & 232 & 238 & 283 & 245 & 268 & 221 & 142 & 247 \\
\hline $\begin{array}{c}\text { UTM } \\
\text { Latitude }\end{array}$ & 49,877 & 49,882 & 49,881 & 49,879 & 49,88 & 49,878 & 49,876 & 49,876 & 49,882 \\
\hline $\begin{array}{c}\text { UTM } \\
\text { Longitude }\end{array}$ & 3,717 & 3,706 & 3,708 & 3,712 & 3,706 & 3,712 & 3,691 & 3,678 & 3,709 \\
\hline
\end{tabular}


182 Modelling, Monitoring and Management of Forest Fires II

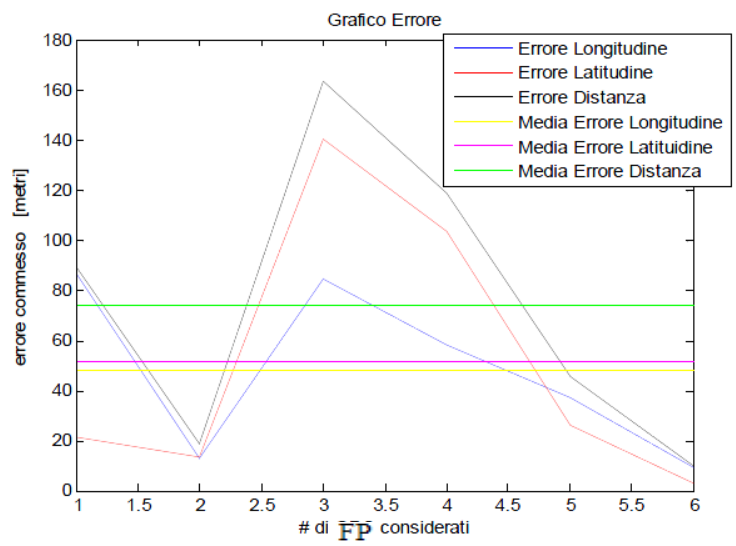

Figure 12: Mismatches in meters between true geographical coordinates and algorithm performance: Longitude (utm), Latitude (utm), distance, and average related them.

Considering decision support, we simulate an event of fire on the monitored scenarios. The algorithm carried out a report, which points out additional support information regarding helicopter landing, water supply positions, intervention squads closer to the fire, fig. 13.

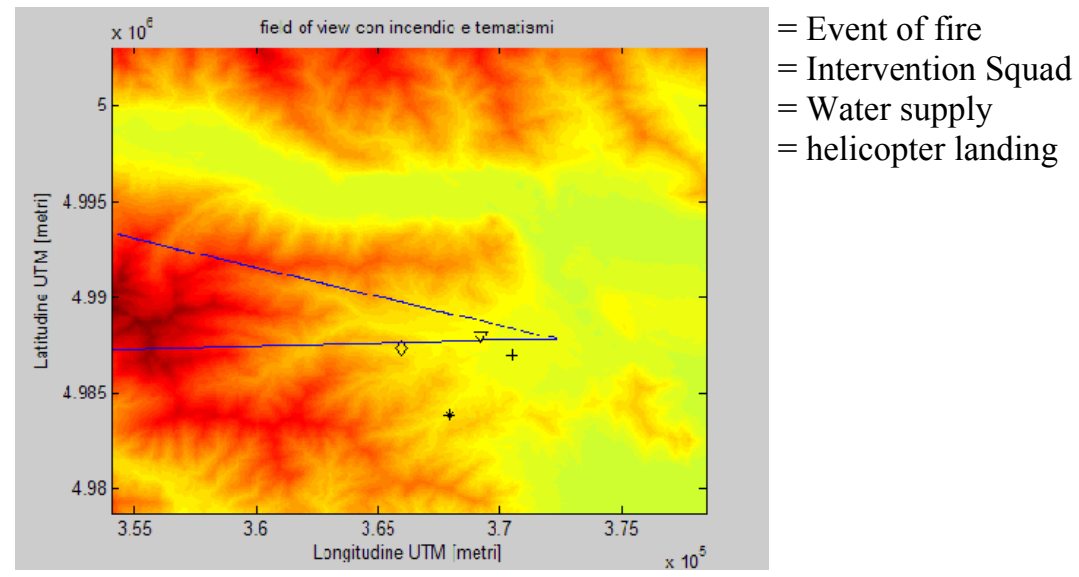

Figure 13: Decision support report on DEM.

\section{Conclusion and future work}

An innovative system based on automatic image geo-referencing methods for forest fire fighting has been implemented. Combining geometric analyses and projective transformations the system can link every single pixel to geographical UTM coordinates within time interval of few seconds with high spatial precision. 
In order to validate the feasibility of the method, a set of images has been analyzed in our test areas. At the moment, our primary target is the successful implementation of the geo-referencing tool using images taken in the visible domain. According to the results shown above, the mismatches between true coordinate and algorithm results is on the order of less hundred meters. In particular, the average longitude error is 50 meters and latitude is more or less 80 meters, on the basis of the spatial resolution of the used DEM. Nevertheless spatial location mismatches are present due to several factors as: DEM and image resolutions, environmental conditions, device sensitivity, fire detection locations and so on. Future target will be spatial error reduction considering larger resolution size of both DEM and images. The algorithm is operating for test activities onboard the integrated monitoring system for high performance decision support SIRIO (Sistema Integrato per il Rilevamento di Incendi bOschivi) developed by EST S.r.1, Remote Sensing Group of Politecnico di Torino and SVM S.r.l.

\section{References}

[1] CORGNATI L.P.; GABELLA M.; PERONA G., FIREcast system Previsional fire danger index computation system for alpine regions, In: Modelling, Monitoring and Management of Forest Fires, 1st International Conference on Forest Fires 2008, Toledo, Spain 17-19 September 2008

[2] Losso A. Corgnati L. Perona G., early forest fires detection: smoke identification through innovative image processing using commercial sensor, Environmental Including Global Change, Palermo, Italy 4-9 Ottobre 2009.

[3] Losso A. Corgnati L. Perona G., false alarm reduction in forest fires detection with low-cost commercial sensors, Gi4DM, Torino, Italy 2-4 Febbraio 2010.

[4] Nurc PO 4050852. Forward Eyes Video Image Rectification and Merging, 31 Dicembre 2005

[5] http://www.photorevolt.com/articoli_id_33.html

[6] Archetti R., Torricelli E., Erdman R., Lamberti A., first application of a new imaging system for the coastal monitoring, Bologna

[7] R. Archetti e A. Lamberti., studio dell'evoluzione di una spiaggia protetta da opera a cresta bassa mediante videomonitoraggio 